\title{
EDITORIAL
}

\section{Vertical disintegration: a strategy for pharmaceutical businesses in 2009?}

\author{
Enhancing the productivity of drug RED might be achieved by vertical disintegration of \\ pharmaceutical business into drug discovery, development and marketing organizations.
}

A recent analysis of the Pharmaprojects database revealed that between 2000 and 2008, 1,941 drugs were discontinued and, where companies disclosed a reason for the discontinuation, in a startling $52 \%$ of cases the reasons were 'strategic' or 'financial'". This obvious misalignment between research decisions and the commercial focus of companies represents a tragic waste of creative talent, intellectual property and, ultimately, the opportunity to improve medical treatments.

Many factors contribute to this misalignment. For example, the genetic complexity of disease and the consequent fragmentation of patient populations mean that the likelihood of finding new blockbuster drugs, where one size fits all, is much smaller than in the past. In addition, the ability of healthcare providers to pay for more stratified medicines if the current costs of drug discovery and development overall (including that of failures and discontinuations) are not reduced is in doubt.

Another important factor is that the efforts of large pharmaceutical companies to satisfy investors' desire for growth have resulted in many product in-licensing deals with smaller companies, often leading to the licensee acquiring the product originator. This removes an independent and potential future supplier of products from the marketplace. Furthermore, experience suggests that the challenges posed by integration of two R\&D organizations often result in the combined organization being less productive, exacerbating the decimation and demoralization of research across the industry.

Finally, from a financial perspective, funders of pharmaceutical businesses do not generally have the 12 to 20 year perspective that is required to translate bioscience ideas into products. Moreover, insufficient overall investment is available today to support the growth of new vertically integrated companies from a research base, as occurred in past decades.

New business models for drug research must therefore be created. We consider that the factors above indicate that the process of creating and selling a drug can no longer be optimally accommodated within one vertically integrated business, and that vertical disintegration into 'discovery', 'development' and 'marketing and sales' businesses is needed.
Discovery organizations would apply their expertise to the translation of research advances into potential drug candidates. For maximum efficiency, resources must be allocated to areas that have the most chance (in the view of expert drug discoverers) of producing a drug candidate, and not be constrained by predicted profitability. These organizations could be funded by a mixture of supragovernment bodies, governments, charities, philanthropists and commercial funders with a perspective that covers both patient interest and the necessary long-term view.

The outputs from these organizations would be developed by a development business most suited to the particular opportunity, which might be commercial or philanthropic. In this respect, the capability of contract research organizations to perform this task could increasingly translate to an equity stake in the product. Finally, a sales and marketing business would license the output from the R\&D organizations. Such a business could effectively balance a portfolio to control its exposure to risk, and although the licenses would be more expensive, the business could be much more confident in its assessment of the marketplace.

Overall, in this model, there is a danger that the new discovery businesses could lack the power that large pharmaceutical company research has harnessed in the past two decades to markedly improve the drug discovery process. Some of the new discovery businesses will have to be large in order to tackle the major drivers of future productivity such as target validation, predictive toxicology and early clinical proof-of-concept. It is also crucial to somehow preserve the traditional role of large pharmaceutical companies in nurturing drug discovery expertise and passing on that skill and knowledge.

Nevertheless, if these challenges can be overcome, this vertical disintegration could allow the three separate businesses to fully exploit their separate capacities, not least because each has access to many suppliers for its inputs and many customers for its outputs. Most importantly, vertical disintegration could result in more - and more appropriate - new medicines in the future.

John Dixon, Geoff Lawton and Peter Machin

1. Biancardi, A. \& Green, S. Scrip 100, S33-S36 (2008). 